\title{
A Redetermination of the Barrier to Internal Rotation of 2-Fluoropropene
}

\author{
G. Bestmann and H. Dreizler \\ Abteilung Chemische Physik im Institut für Physikalische Chemie der Universität Kiel \\ Z. Naturforsch. 40 a, 267-270 (1985); received January 15, 1985

\begin{abstract}
We present the determination of the methyl barrier to internal rotation from the rotational spectrum in the ground state of 2-fluoropropene. A complete set of barrier parameters could be determined. The results are compared with those of 2-chloro- and 2-bromopropene, achieved by the same technique and evaluation method.
\end{abstract}

Recently we determined the barrier to internal rotation of the methyl group from rotational transitions in the torsional ground state of 2-chloro- and 2 -bromopropene $[1,2]$. Now we present a redetermination of the barrier to internal rotation of 2 -fluoropropene. The measurements were made by microwave Fourier transform spectroscopy [3-6] because of the higher resolution compared to Stark spectroscopy.

2-fluoropropene, $\mathrm{CH}_{3}-\mathrm{CF}=\mathrm{CH}_{2}$, was first investigated by Pierce and O'Reilly [7]. The substance was purchased from PCR Research Chemicals, Gainesville, Florida, USA, and used after vacuum distillation. The spectra between 8 and $26 \mathrm{GHz}$ were recorded in the pressure range between 1 to $2 \mathrm{mTorr}$ and at a temperature of approximately $-30^{\circ} \mathrm{C}$. Our measurements are given in Table 1 . We performed a sixth order centrifugal distortion analysis of $83 \mathrm{~A}$ species lines with a standard deviation of $5 \mathrm{kHz}$. The Hamiltonian of van Eijck [8] and Typke [9] (Eq. (6)) was used. It is worth to mention, that for 2-bromopropene only one centrifugal distortion parameter $\delta_{J}^{\prime}$ could be determined. In 2-chloropropene a fourth order analysis was possible. The results for 2-fluoropropene are given in Table 2.

The barrier $V_{3}$ was determined from $77 \mathrm{~A}-\mathrm{E}$ splittings given in Table 1 . The closely split doublets were corrected for effects of overlapping by a line shape analysis [10]. The internal axis method (IAM) was applied with a version of Wood's program [11-14]. Using the rotational constants of Table 2 a fit of the Fourier coefficient $w_{1}(s), s$ the reduced

Reprint requests to Prof. Dr. H. Dreizler, Institut für Physikalische Chemie der Universität Kiel, Olshausenstr. 40, D-2300 Kiel.
Table 1. Measured transitions of 2-fluoropropene $\mathrm{CH}_{3}-\mathrm{CF}=$ $\mathrm{CH}_{2} . \Gamma$ : symmetry species, $v_{\mathrm{m}}$ : measured frequency, deviation from calculated frequency in brackets in units of the last digit, $\Delta v_{\mathrm{m}}$ : measured internal rotation splitting $v_{\mathrm{A}}-v_{\mathrm{E}}$, $\Delta v_{\mathrm{c}}$ : calculated internal rotation splitting, all frequencies in [MHz]. (****: not resolved).

\begin{tabular}{|c|c|c|c|c|c|c|c|c|c|}
\hline$J$ & $K_{-}$ & & $J^{\prime}$ & $K_{-}^{\prime}$ & $K_{+}^{\prime}$ & $\Gamma$ & $v_{\mathrm{m}}$ & $\Delta v_{\mathrm{m}}$ & $\Delta v_{\mathrm{c}}$ \\
\hline 1 & 0 & 1 & $\circ$ & 0 & 0 & $\stackrel{A}{E}$ & $13968.700(-4)$ & $* * * * *$ & 0.000 \\
\hline 1 & 1 & 1 & 0 & 0 & 0 & $\begin{array}{l}A \\
E\end{array}$ & $15096.752(0)$ & 0.046 & 0.047 \\
\hline 2 & 0 & 2 & 1 & 0 & 1 & $\stackrel{A}{E}$ & $24749.032(2)$ & 0.031 & 0.031 \\
\hline 2 & 1 & 2 & 1 & 1 & 1 & $\stackrel{A}{E}$ & 23822.771 (3) & $* * * * *$ & 0.000 \\
\hline 2 & o & 2 & 1 & 1 & 1 & $\begin{array}{l}A \\
E\end{array}$ & $23620.977(2)$ & $* * * * *$ & -0.015 \\
\hline 2 & 1 & 2 & 1 & 0 & 1 & $\begin{array}{l}A \\
E\end{array}$ & $24950.833(-4)$ & 0.054 & 0.047 \\
\hline 2 & 1 & 1 & 2 & 1 & 2 & $\stackrel{A}{E}$ & $12343.558(5)$ & $* * * * *$ & 0.000 \\
\hline 2 & 2 & 1 & 2 & 0 & 2 & $\begin{array}{l}A \\
E\end{array}$ & $15929.497(-11)$ & 0.167 & 0.156 \\
\hline 2 & 1 & 1 & 2 & 0 & 2 & $\stackrel{A}{E}$ & 12545.348 (11) & $* * * * *$ & 0.017 \\
\hline 2 & 2 & 1 & 2 & 1 & 2 & $\begin{array}{l}A \\
E\end{array}$ & $15727.691(0)$ & 0.144 & 0.140 \\
\hline 3 & 1 & 3 & 2 & 2 & 0 & $\begin{array}{l}A \\
E\end{array}$ & $15208.314(-1)$ & -0.103 & -0.095 \\
\hline 3 & 1 & 2 & 3 & 1 & 3 & $\stackrel{A}{E}$ & $22539.450(-4)$ & 0.060 & 0.055 \\
\hline 3 & 2 & 1 & 3 & 2 & 2 & $\begin{array}{l}A \\
E\end{array}$ & $10790.888 \quad(7)$ & -0.061 & -0.057 \\
\hline 3 & 2 & 2 & 3 & 0 & 3 & $\stackrel{A}{E}$ & $23532.045(-1)$ & 0.131 & 0.129 \\
\hline 3 & 3 & 1 & 3 & 1 & 2 & $\begin{array}{l}A \\
E\end{array}$ & $18505.639(-2)$ & 0.287 & 0.288 \\
\hline 3 & 3 & 0 & 3 & 2 & 1 & $\begin{array}{l}A \\
E\end{array}$ & $8892.587(-6)$ & 0.230 & 0.221 \\
\hline 3 & 1 & 2 & 3 & 0 & 3 & $\stackrel{A}{E}$ & $22562.448(-4)$ & 0.064 & 0.057 \\
\hline 3 & 2 & 1 & 3 & 1 & 2 & $\stackrel{A}{E}$ & $11760.487(8)$ & 0.011 & 0.015 \\
\hline & 2 & 2 & 3 & 1 & 3 & $\begin{array}{l}A \\
E\end{array}$ & $23509.053(-7)$ & 0.133 & 0.127 \\
\hline
\end{tabular}

0340-4811/85/0300-0267\$01.30/0. - Please order a reprint rather than making your own copy. 
Table 1 (continued)

\begin{tabular}{lllllllllll}
\hline$J$ & $K_{-}$ & $K_{+}$ & $J^{\prime}$ & $K_{-}^{\prime}$ & $K_{+}^{\prime}$ & $\Gamma$ & $v_{\mathrm{m}}$ & $\Delta v_{\mathrm{m}}$ & $\Delta v_{\mathrm{c}}$ \\
\hline 3 & 3 & 1 & 3 & 2 & 2 & $E_{E}^{A}$ & $17536.043(-6)$ & 0.228 & 0.216
\end{tabular}

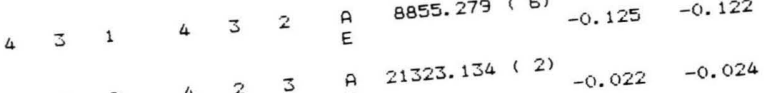

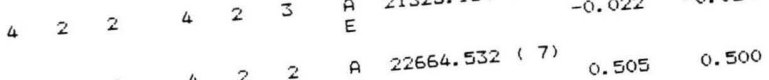

$\begin{array}{lllllllllll}4 & 4 & 1 & 4 & 2 & 2 & A & & & & \\ & & & & & & E & & & & \\ 4 & 3 & 2 & 4 & 1 & 3 & A & 24168.354 & (-1) & 0.178 & 0.179\end{array}$

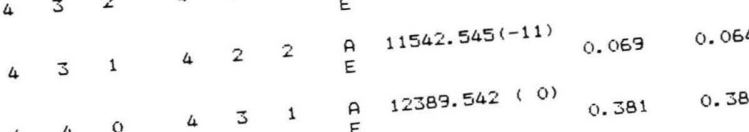

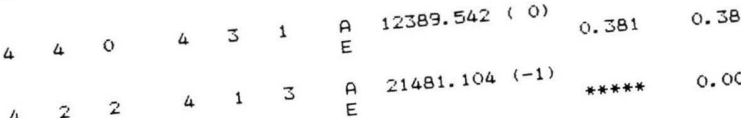

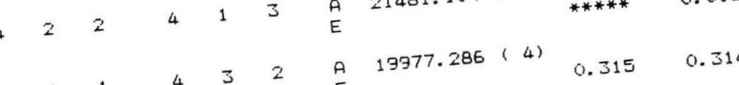

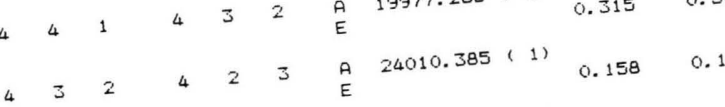

$5=3253 \quad \begin{array}{lllllll}A & 19369.057(-1) & -0.132 & -0.129\end{array}$

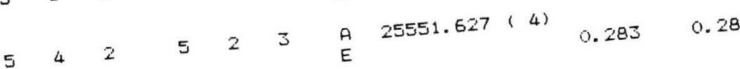

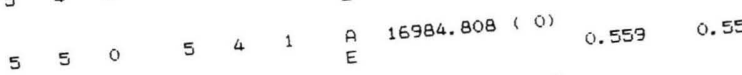

$532523 \quad \begin{array}{lllllll}A & 19977.043(1) & -0.073 & -0 & & & \end{array}$

$\begin{array}{llllllllll}5 & 4 & 1 & 5 & 3 & 2 & A & 12291.698 & (-5) & 0.181\end{array}$

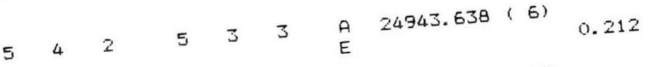

$5 \quad 5 \quad 1 \quad 55 \quad 4 \quad 2 \quad A$

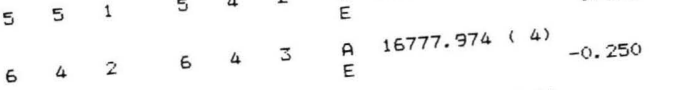

$\begin{array}{lllllllllll}A & 5 & 1 & 6 & 4 & 2 & E^{2} & 14285.794 & (1) & 0.352\end{array}$

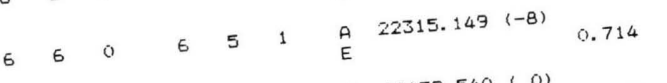

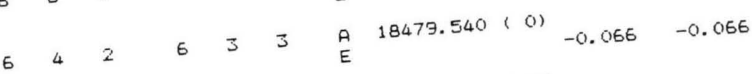

$\begin{array}{lllllllllll}6 & 5 & 2 & 6 & 4 & 3 & A & 26421.425 & (3) & 0.303 & 0.312\end{array}$

$\begin{array}{lllllll}7 & 5 & 2 & 7 & 5 & 3 & \mathrm{~A}\end{array}$

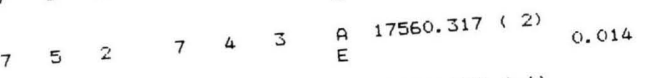

$\begin{array}{llllllllll}7 & 5 & 1 & 7 & 5 & 2 & A & 17668.868 & (-1) & 0.591\end{array}$

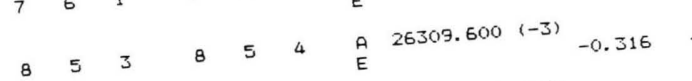

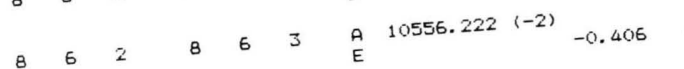

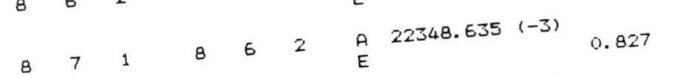

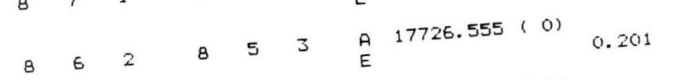

$\begin{array}{lllllllllllll}A & 6 & 3 & 9 & 6 & 4 & E^{2} & & & & & & \end{array}$

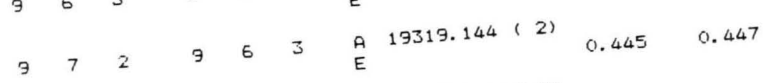

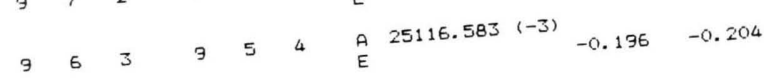

Table 1 (continued)

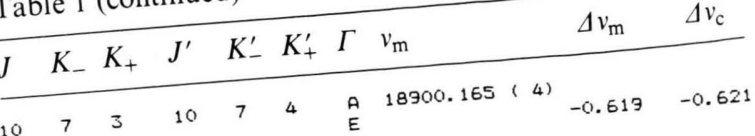

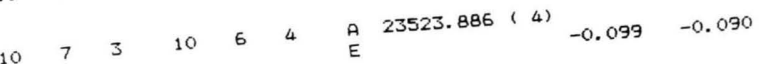
$\begin{array}{llllllllll}A & 2 & 10 & 7 & 3 & E^{2} & 22497.024 & (4) & 0.754 & 0.757\end{array}$

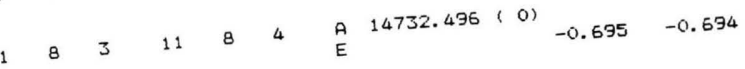
$\begin{array}{lllllllllll}1 & 8 & 3 & 11 & 7 & 4 & A^{2} & 23072.795 & (-8) & 0.154 & 0.146\end{array}$

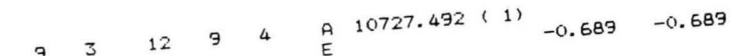
$\begin{array}{llllllllllll}12 & 9 & 3 & 12 & 8 & 4 & A & 24182.945 & (3) & 0.477 & 0.482\end{array}$

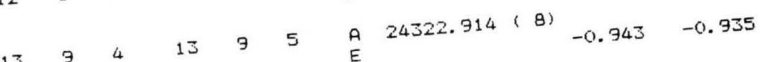
$\begin{array}{lllllllll} & 14 & 10 & 5 & A & 19253.228 & (4) & -1.022 & -1.016\end{array}$ $15114 \quad 1511 \quad 5 \quad \frac{A}{E} 14325.285(1)-0.998-0.995$ $\begin{array}{llllllllllll}16 & 12 & 4 & 16 & 12 & 5 & A & 9950.055 & (-2) & -0.873 & -0.873\end{array}$ $\begin{array}{lllllllllll}17 & 12 & 5 & 17 & 12 & 6 & A_{E} & 24117.334 & (-2) & -1.330 & -1.339\end{array}$ $\begin{array}{lllllllllll}18 & 13 & 5 & 18 & 13 & 6 & A & 18294.926 & (-4) & -1.296 & -1.306\end{array}$ $\begin{array}{llllllllll}19 & 5 & 19 & 14 & 6 & A & 13019.608 & (-1) & -1.153 & -1.151\end{array}$

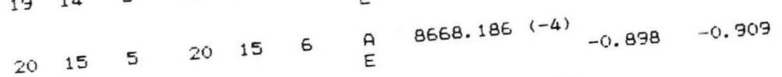

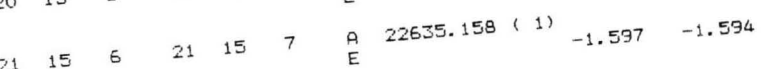
$\begin{array}{llllllllllll}22 & 16 & 6 & 22 & 16 & 7 & A & 16460.846 & (0) & & & \end{array}$ $\begin{array}{lllllllllll}23 & 17 & G & 23 & 17 & 7 & A & 11235.556 & (-2) & -1.137 & -1.133\end{array}$

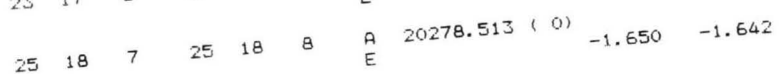
$\begin{array}{llllllllllll}26 & 19 & 7 & 26 & 19 & 8 & A & 14157.112(2) & -1.310 & -1.332\end{array}$ $\begin{array}{llllllllllll}27 & 20 & 7 & 27 & 20 & 8 & A & 9307.388 & (-2) & 0.977 & -0.976\end{array}$ $\begin{array}{lllllllllll}28 & 20 & 8 & 28 & 20 & 9 & A & 24475.415 & (-6) & -1.812 & -1.814\end{array}$

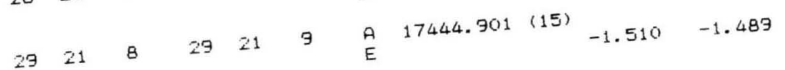
$\begin{array}{llllllllllll}30 & 22 & 8 & 30 & 22 & 9 & A & 11726.740 & (0) & -1.108 & -1.108\end{array}$

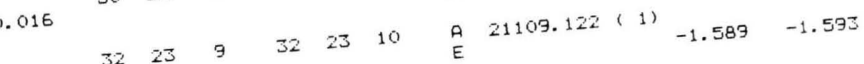
0.588 0.325

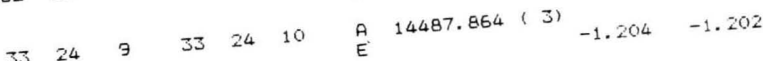

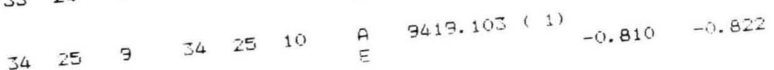

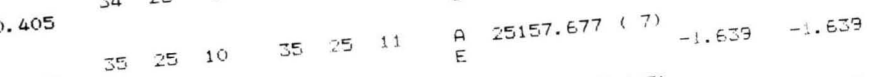
$\begin{array}{lllllllll}36 & 26 & 11 & A & 17607.977 & (7) & -1.252 & -1.251\end{array}$

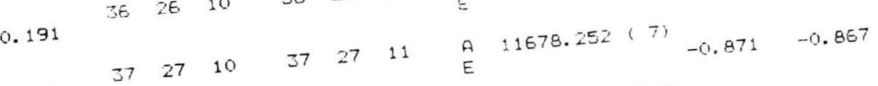

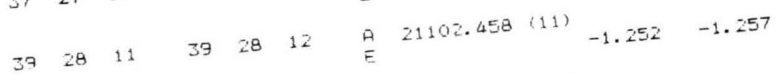
\begin{tabular}{lllllll}
40 & 29 & 11 & 40 & 29 & 12 & $A$ \\
\hline
\end{tabular} 
Table 2. Rotational and centrifugal distortion constants of 2-fluoropropene $\mathrm{CH}_{3}-\mathrm{CF}=\mathrm{CH}_{2}$. $\sigma$ : standard deviation of the fit, standard errors in units of the last digit in brackets.

$A=10169.70848(108) \mathrm{MHz}$
$B=9041.63008(109) \mathrm{MHz}$
$C=4927.06935(109) \mathrm{MHz}$

$$
\begin{aligned}
& D_{J}^{\prime}=2.440(89) \mathrm{kHz} \\
& D_{J K}^{\prime}=12.997(13) \mathrm{kHz} \\
& D_{K}^{\prime}=-4.813(9) \mathrm{kHz} \\
& \delta_{J}^{\prime}=1.643(12) \mathrm{kHz} \\
& R_{6}^{\prime}=-0.830(6) \mathrm{kHz} \\
& \sigma=5 \mathrm{kHz}
\end{aligned}
$$

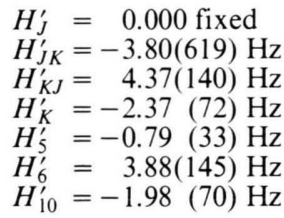

$H_{J}^{\prime}=0.000$ fixed

$H_{J K}=-3.80(619) \mathrm{Hz}$

$H_{K J}^{\prime}=4.37(140) \mathrm{Hz}$

$H_{5}^{\prime}=-0.79(33) \mathrm{Hz}$

$H_{10}^{\prime}=-1.98(70) \mathrm{Hz}$

Correlation coefficients

\begin{tabular}{rrrrrrrrrrrrr}
1.00 & & & & & & & & & & & & \\
0.99 & 1.00 & & & & & & & & & & \\
0.91 & 0.92 & 1.00 & & & & & & & & & \\
0.85 & 0.86 & 0.89 & 1.00 & & & & & & & & \\
0.11 & 0.17 & -0.09 & 0.04 & 1.00 & & & & & & & \\
0.08 & 0.02 & -0.06 & -0.05 & -0.46 & 1.00 & & & & & & \\
0.13 & 0.18 & -0.10 & 0.05 & 0.89 & -0.07 & 1.00 & & & & & \\
0.10 & 0.15 & -0.07 & 0.07 & 0.88 & -0.16 & 0.98 & 1.00 & & & & \\
-0.08 & -0.02 & 0.06 & 0.09 & 0.42 & -0.48 & 0.40 & 0.56 & 1.00 & & & \\
0.08 & 0.01 & -0.06 & -0.08 & -0.38 & 0.44 & -0.37 & -0.53 & -0.99 & 1.00 & & \\
-0.08 & 0.01 & 0.05 & 0.07 & 0.32 & -0.32 & 0.35 & 0.49 & 0.92 & -0.97 & 1.00 & \\
-0.03 & 0.01 & 0.04 & 0.09 & 0.51 & -0.35 & 0.56 & 0.70 & 0.95 & -0.91 & 0.82 & 1.00 & \\
0.06 & 0.01 & -0.06 & -0.09 & -0.44 & 0.45 & -0.45 & -0.60 & -0.99 & 0.97 & -0.90 & -0.98 & 1.00 \\
-0.09 & -0.02 & 0.07 & 0.08 & 0.38 & -0.51 & 0.34 & 0.50 & 0.99 & -0.99 & 0.93 & 0.92 & -0.98 \\
\hline
\end{tabular}

Table 3. Internal rotation parameters of 2-fluoropropene, $\mathrm{CH}_{3}-\mathrm{CF}=\mathrm{CH}_{2} . w_{1}(s)$ : Fourier coefficient, $\Varangle(a, i)$ : angle between the inertia axis $a$ and the internal rotation axis $i$, $I_{\alpha}$ : moment of inertia of the methyl group, $(\cdots)$ : correlation coefficient, $\sigma$ : standard deviation of the fit, $\Delta v$ : mean experimental splitting, $s$ : reduced barrier height, $F$ : reduced rotational constant of the internal rotation, $V_{3}$ : barrier to internal rotation, $N$ : Number of splittings, standard errors in units of the last digit in brackets.

\begin{tabular}{lc}
\hline$w_{1}(s)$ & $-0.9256(93) \cdot 10^{-5}$ \\
$\nless(a, i)\left[{ }^{\circ}\right]$ & $5.84(75)$ \\
$I_{\alpha}\left[\mathrm{amu} \AA^{2}\right]$ & $3.177(18)$ \\
$\left(w_{1}(s), \nless(a, i)\right)$ & -0.995 \\
$\left(w_{1}(s), I_{\alpha}\right)$ & 0.970 \\
$\left(\nless(a, i), I_{\alpha}\right)$ & -0.980 \\
$\sigma[\mathrm{kHz}]$ & 7 \\
$\Delta v[\mathrm{kHz}]$ & 583 \\
$s$ & $65.41(9)$ \\
$F[\mathrm{GHz}]$ & $169.929(96)$ \\
$V_{3}[\mathrm{kcal} / \mathrm{mol}]$ & $2.384(17)$ \\
$N$ & 77 \\
\hline
\end{tabular}

barrier, $\Varangle(a, i)$, the angle between the inertia axis $a$ and the internal rotation axis $i$ and $I_{\alpha}$, the moment of inertia of the methyl group, converged to reasonable values as given in Table 3 . For this molecule the value of $I_{\alpha}=3.177(18) \mathrm{amu} \AA^{2}$ could be fitted, which is near to the value of $I_{\alpha}=$ $3.167 \mathrm{amu} \AA^{2}$ for 2-chloropropene derived in [1] from a complete $r_{\mathrm{s}}$-structure [15].

Table 5 of [2] may now be completed with measurements taken by the same technique and evaluated by the same method. The comparison is given in Table 4. The general trend in variation of the barrier height $V_{3}$ is like for $\mathrm{CH}_{3} \mathrm{CH}_{2} \mathrm{X}, \mathrm{X}=\mathrm{F}, \mathrm{Cl}$, and $\mathrm{Br}$. $V_{3}$ increases from $\mathrm{F}$ to $\mathrm{Cl}$ and $\mathrm{Br}$.

We thank the members of our group for help, the Deutsche Forschungsgemeinschaft and Fonds der Chemie for funds. The calculations were made at the computer center of the University of Kiel.

Table 4. Comparison of potential barriers $V_{3}$ and related parameters of 2-halopropenes $\mathrm{CH}_{3}-\mathrm{CX}=\mathrm{CH}_{2}, \mathrm{X}=\mathrm{F}, \mathrm{Cl}$ and Br. Assumptions in square brackets.

\begin{tabular}{llcccc}
\hline $\mathrm{X}$ & $\mathrm{F}$ & ${ }^{35} \mathrm{Cl}$ & ${ }^{37} \mathrm{Cl}$ & ${ }^{79} \mathrm{Br}$ & ${ }^{81} \mathrm{Br}$ \\
\hline$V_{3}[\mathrm{cal} / \mathrm{mol}]$ & $2384(17)$ & $2568(10)$ & $2582(12)$ & $2571(49)$ & $2558(74)$ \\
$\nless(a, i)\left[{ }^{\circ}\right]$ & $5.84(75)$ & $59.78(84)$ & $61.28(108)$ & $56.8(34)$ & $56.9(48)$ \\
$I_{\alpha}\left[\mathrm{amu} \AA^{2}\right]$ & $3.177(18)$ & {$[3.167]$} & {$[3.167]$} & {$[3.167]$} & {$[3.167]$} \\
$F[\mathrm{GHz}]$ & $169.93(96)$ & 165.91 & 165.69 & 164.64 & 164.72 \\
$s$ & $65.41(9)$ & $72.15(29)$ & $72.63(35)$ & $72.8(14)$ & $72.4(21)$ \\
& this work & {$[1]$} & {$[1]$} & {$[2]$} & {$[2]$} \\
\hline
\end{tabular}


[1] E. Fliege and H. Dreizler, Z. Naturforsch. 38 a, 1231 (1983).

[2] E. Fliege and H. Dreizler, Z. Naturforsch. 39a, 637 (1984).

[3] G. Bestmann, H. Dreizler, H. Mäder, and U. Andresen, Z. Naturforsch. 35a, 392 (1980).

[4] G. Bestmann and H. Dreizler, Z. Naturforsch. 37a, 58 (1982).

[5] G. Bestmann, H. Dreizler, E. Fliege, and W. Stahl, J. Mol. Struct. 97, 215 (1983).

[6] W. Stahl, E. Fliege, H. Dreizler, and R. Schwarz, Z. Naturforsch. 39 a, 354 (1984), and to be published.

[7] L. Pierce and J. M. O'Reilly, J. Mol. Spectr. 3, 536 (1959).
[8] B. P. van Eijck, J. Mol. Spectr. 53, 246 (1974).

[9] V. Typke, J. Mol. Spectr. 63, 170 (1976); program ZFAP6 by V. Typke, 1982

[10] E. Fliege and H. Dreizler, Z. Naturforsch. 39a, 630 (1984).

[11] R. C. Woods, J. Mol. Spectr. 21, 4 (1966).

[12] R. C. Woods, J. Mol. Spectr. 22, 49 (1967).

[13] J. Meier, Dissertation 4611, ETH Zürich, 1970.

[14] Modified by H. Lutz, E. Fliege, and G. Bestmann; programs IAMCAL, IAMFIT and IAMMOD.

[15] W. Good, R. J. Cohan, jr., A. Bauder, and Hs. H. Günthard, J. Mol. Spectr. 41, 381 (1972). 